\title{
Fatal Meningitis in Patient with X-Linked Chronic Granulomatous Disease Caused by Virulent Granulibacter bethesdensis
}

\section{Mafalda Rebelo, ${ }^{1}$ Li Ding, ${ }^{1}$ Ana Isabel Cordeiro, Conceição Neves, Maria João Simões, Adrian M. Zelazny, Steven M. Holland, ${ }^{2}$ João Farela Neves ${ }^{2}$}

Granulibacter bethesdensis is a pathogen reported to cause recurrent lymphadenitis exclusively in persons with chronic granulomatous disease. We report a case of fatal meningitis caused by a highly virulent $G$. bethesdensis strain in an adolescent in Europe who had chronic granulomatous disease.

$\mathrm{C}$ hronic granulomatous disease (CGD) is a primary immunodeficiency characterized by a deficient nicotinamide adenine dinucleotide phosphate oxidative burst that impairs phagocyte superoxide formation and killing of certain pathogens. Mutations can occur in any of the 5 subunits of nicotinamide adenine dinucleotide phosphate oxidase. Most cases are inherited as X-linked defects (gp$91^{\text {phox }}$ ), but they also can occur in an autosomal recessive manner (1). Increased susceptibility develops to recurrent infections of the skin, lymph nodes, lungs, and other organs (2), mostly caused by bacteria and fungi, including Staphylococcus aureus, Serratia marcescens, Burkholderia cepacia, Salmonella spp., Nocardia spp., and Aspergillus spp. (2). Emerging organisms, such as Granulibacter bethesdensis and other methylotrophs, occur almost exclusively in CGD patients $(3,4)$.

G. bethesdensis was first described in 2006, when it was isolated in a CGD patient with lymphadenitis (4). It is a gram-negative, aerobic, oxidase-negative, catalasepositive, nonmotile coccobacillus to rod-shaped bacterium belonging to the Acetobacteraceae family $(5,6)$. G. bethesdensis was the first of these Acetobacteraceae

Author affiliations: Hospital Dona Estefânia-Centro Hospitalar de Lisboa Central, Entidade Publica Empresarial, Lisbon, Portugal

(M. Rebelo, A.I. Cordeiro, C. Neves, J.F. Neves); National Institute of Allergy and Infectious Diseases of the National Institutes of Health, Bethesda, Maryland, USA (L. Ding, A.M. Zelazny,

S.M. Holland); National Institute of Health Dr. Ricardo Jorge,

Lisbon (M.J. Simões); Chronic Diseases Research Center, NOVA

Medical School, Lisbon (J.F. Neves)

DOI: https://doi.org/10.3201/eid2505.181505 family bacteria with proven pathogenicity in humans, causing invasive disease in CGD patients and mice (4). It has been mostly linked to indolent nonfatal lymphadenitis and deep neck infections in patients in North America. The infection can recur over several years by reactivation of the same strain or reinfection with different strains $(3,7-9)$. The first fatal infection was reported in a 10-yearold boy from Spain, who died of fulminant sepsis (10). In vitro, $G$. bethesdensis shows extensive resistance to various antimicrobial drugs, although its slow growth makes susceptibility testing difficult. Ceftriaxone, aminoglycosides, doxycycline, and trimethoprim/sulfamethoxazole showed activity in vitro (7).

We report a case of $G$. bethesdensis meningitis in a patient with X-linked CGD. We also report animal data comparing this $G$. bethesdensis strain with the strain recovered from recurrent lymphadenitis in a US CGD patient.

\section{The Study}

The patient was a 16-year-old boy whose X-linked CGD (CYBB exon 13 deletion) was diagnosed when he was 2 years old. His disease had been well-controlled with cotrimoxazole, itraconazole, and interferon- $\gamma$. In September 2014, he was hospitalized with a deep cervical abscess (Figure 1, panel A) and received a 5-week course of intravenous ciprofloxacin, doxycycline, and ceftriaxone that resulted in complete clinical and radiologic resolution, followed by 6 weeks of oral amoxicillin/clavulanic acid, doxycycline, and ciprofloxacin along with his usual prophylaxis. No pathogen was identified despite blood cultures, bronchoalveolar lavage, and lymph node biopsy cultures and broad-range bacterial PCR.

After this regimen was completed, the boy was readmitted for 8 weeks with pneumonia with pleural effusion (Figure 1, panel B). Full 16S rRNA gene sequencing $(\approx 1,500$ bp) identified Cupriavidus spp. in pleural fluid. He received meropenem, amikacin, ciprofloxacin, teicoplanin, doxycycline, and voriconazole, and his condition improved. However, 2 weeks later, fever returned, along with splenomegaly, hemodynamic instability, pancytopenia,

${ }^{1}$ These authors contributed equally to this article.

${ }^{2}$ These authors contributed equally to this article. 

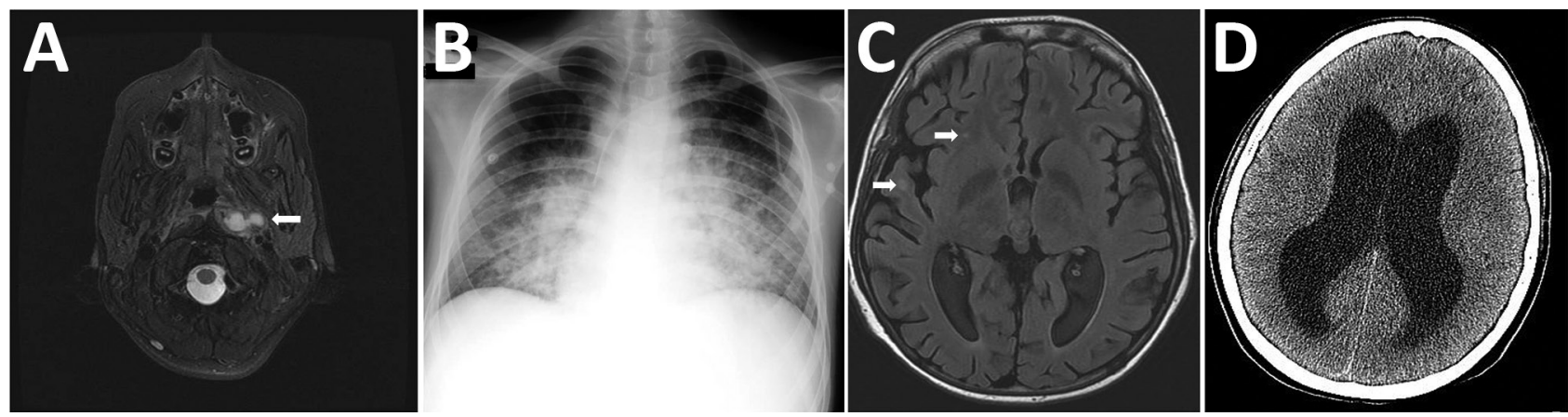

Figure 1. Most relevant imaging results from a 16-year-old boy with X-linked chronic granulomatous disease who died of meningitis caused by a virulent Granulibacter bethesdensis strain. A) Computed tomography image showing deep cervical abscess (arrow), July 2012. B) Radiograph showing pneumonia with pleural effusion, October 2012. C) Cranial magnetic resonance image showing multiple intraparenchymal brain abscesses (arrows), December 2012. D) The patient died of obstructive hydrocephalus (shown) and multiorgan failure, April 2013.

hypofibrinogenemia, hyperferritinemia, and elevated soluble CD25. He received intravenous immunoglobulin and dexamethasone for this inflammatory condition and fully recovered. Neck and lung computed tomography images and positron emission tomography performed 1 month later showed no signs of active infection.
Nevertheless, a few days later, the patient sought care for altered mental status, hallucinations, aggressiveness, and respiratory instability requiring admission to the pediatric intensive care unit. He had extensive bilateral pneumonia and multiple intraparenchymal brain abscesses (Figure 1, panel C). Meropenem, ciprofloxacin, amikacin, doxycycline,
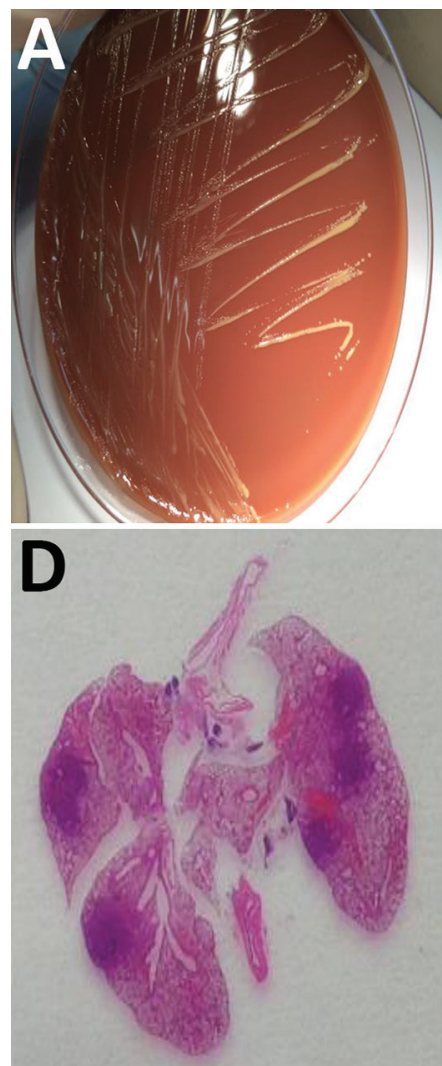
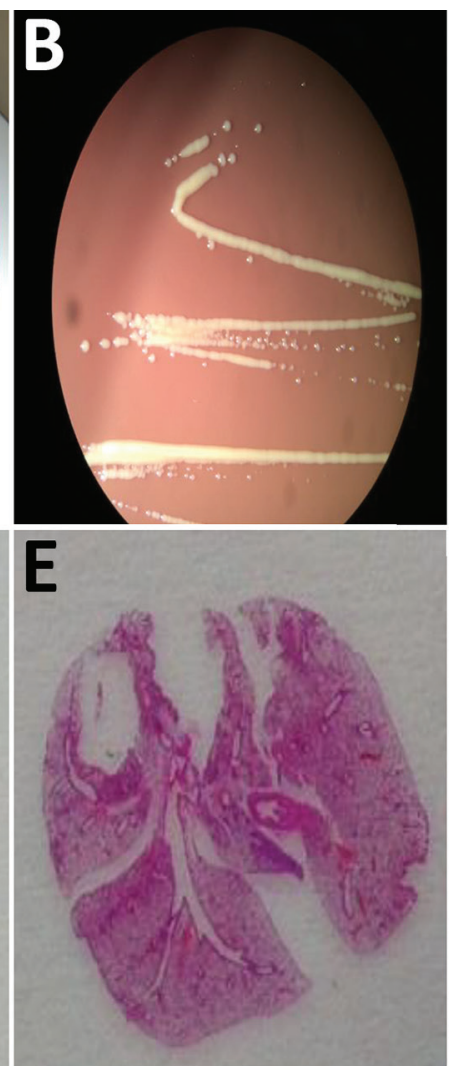
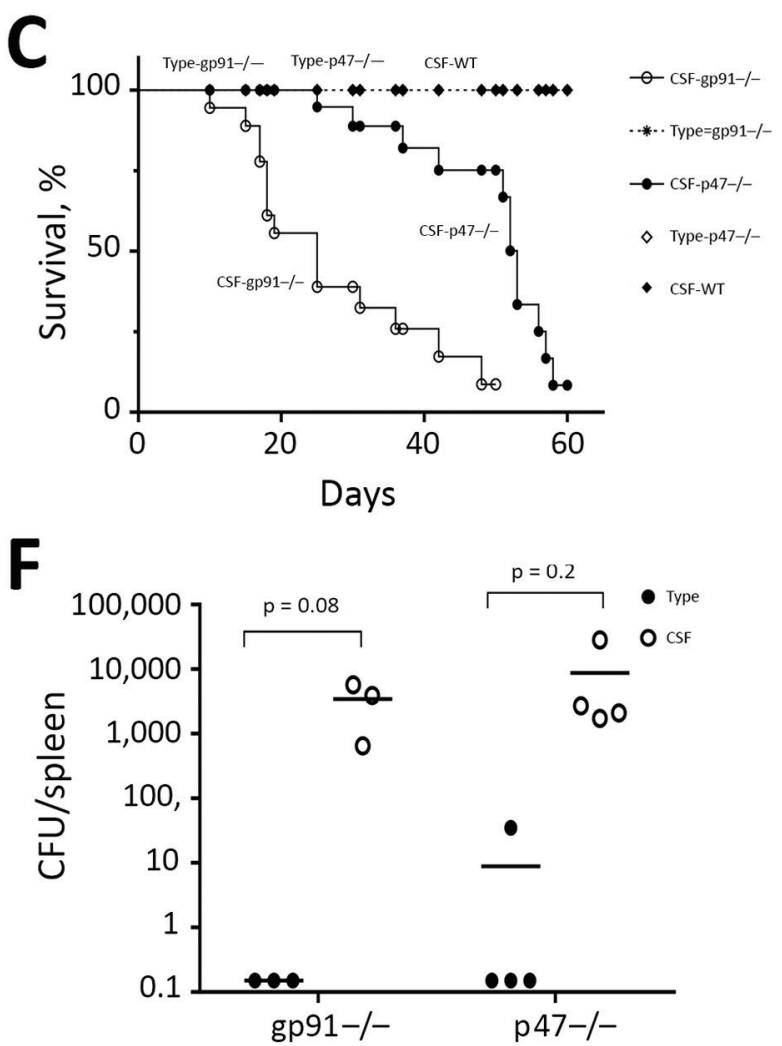

Figure 2. Granulibacter bethesdensis colonies and pathology results after inoculation on mouse models. A, B) CSF on chocolate agar for 4 days showed slowly growing brown-yellow, shiny colonies $2-4 \mathrm{~mm}$ in diameter. Full $16 \mathrm{~S}$ RNA sequencing $(\approx 1,500 \mathrm{bp})$ led to identification of $G$. bethesdensis (with $99.7 \%$ match). C) Survival of gp91/p47 KO mice after inoculation of different G. bethesdensis strains. D) Pathology images of gp91 KO mouse lung after CSF strain infection. E) Pathology images of gp91 KO mouse lungs after type strain infection. F) Quantification of G. bethesdensis strains in spleens of gp91/p47 KO mice after inoculation. CSF, cerebrospinal fluid; type, National Institutes of Health type strain; WT, wild type. 
Table. Bacterial culture from blood and brain samples of gP91 KO mouse infected with Granulibacter bethesdensis after 4 weeks* $^{*}$

\begin{tabular}{|c|c|c|c|}
\hline \multirow[b]{2}{*}{ Mouse } & \multirow[b]{2}{*}{ Strain } & \multicolumn{2}{|c|}{ Sample } \\
\hline & & Blood & Brain \\
\hline 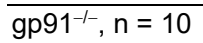 & Type, USA & 0 & 0 \\
\hline $\mathrm{gp} 91^{-1-}, \mathrm{n}=10$ & CSF, Portugal & 5 & 2 \\
\hline $\mathrm{p} 47^{-1-}, \mathrm{n}=5$ & Type & 0 & 0 \\
\hline $\mathrm{p} 47^{-l-}, \mathrm{n}=5$ & CSF & 0 & 0 \\
\hline WT, $n=9$ & CSF, Portugal & 0 & ND \\
\hline $\begin{array}{l}{ }^{*} \text { Bold indicate } \\
\text { mouse. CSF, }\end{array}$ & $\begin{array}{l}\text { ts of the inoculation } \\
\text { nal fluid; ND, not d }\end{array}$ & air & $91 \mathrm{KO}$ \\
\hline
\end{tabular}

teicoplanin, and voriconazole were started; results of cerebrospinal fluid (CSF) and lung biopsy samples were unremarkable. Teicoplanin was switched to linezolid and voriconazole to caspofungin and liposomal amphotericin B because of toxicity concerns. Four weeks later, he was discharged from the intensive care unit. One month later, fever, vomiting, and focal neurologic deficits developed. CSF showed pleocytosis and hypoglycorrhachia with elevated protein levels. Cerebral imaging confirmed leptomeningitis. Isoniazid, clarithromycin, and rifampin were added to his treatment regimen, but his neurologic status continued to deteriorate. Obstructive hydrocephalus (Figure 1, panel D) and multiorgan failure developed, and he died 3 months later.

CSF cultures yielded yellow-brown, shiny, small colonies $(2-4 \mathrm{~mm})$ on chocolate agar after 4 days' incubation. (Figure 2, panels A, B). Full 16S RNA sequencing $(\approx 1,500 \mathrm{bp})$ showed $99.7 \%$ match to the type strain of G. bethesdensis CGDNIH1T (ATCC BAA-1260T, DSM $17861 \mathrm{~T}$ ) from North America and 100\% match to a previously reported G. bethesdensis strain from Spain. Nonstandardized susceptibility test using Etest, performed in Mueller Hinton agar supplemented with 5\% sheep blood agar with an overnight air incubation at $37^{\circ} \mathrm{C}$, showed resistance to doxycycline (MIC $24 \mathrm{mg} / \mathrm{L}$ ) and ceftriaxone ( $\mathrm{MIC}>32 \mathrm{mg} / \mathrm{L}$ ).

We used mouse models of CGD to determine whether differences existed in immune response, pathogenicity, or severity of disease between the European (CSF strain) and the US (type strain) strains. We intraperitoneally infected gp91 phox-/- mice with $10^{7} \mathrm{CFU}$ of $G$. bethesdensis type strain and $\mathrm{p} 47^{\text {phox-1- }}$ mice intraperitoneally with $10^{7} \mathrm{CFU}$ of CSF strain and monitored moribundity during infection. We euthanized gp $91^{\text {phox-1- }}$ mice 4 weeks and p47 $7^{\text {phox-/- }}$ mice 8 weeks after infection and collected brain, spleen, lung, lymph nodes, and blood for culture, bacterial enumeration, and histopathologic examination. Plasma cytokines were assayed.

Although we observed differences between gp91 $1^{\text {phox }-/-}$ and $\mathrm{p} 47^{\text {pho- }-}$ mice, both CGD mice models showed high rates of death when infected with CSF strain. No deaths occurred in mice infected with type strain, nor did CSF strain cause disease in wild-type mice (Figure 2, panel C). CSF strain-infected mice showed more severe pathologic organ changes than did type strain-infected mice (Figure 2, panels D, E; Appendix Figure 1, https://wwwnc.cdc.gov/ EID/article/25/5/18-1505-App1.pdf). We performed quantitative cultures to assess bacterial load in the spleens of inoculated mice. CSF strain CFUs were 100-1,000 times higher than those of type strain 4 and 8 weeks after infection. (Table; Figure 2, panel F). In addition, infection with CSF strain yielded 100-1,000 times higher CFUs in spleens of CGD mice than in wild-type mice 4 and 8 weeks after infection. (Appendix Figure 2). CSF strain showed faster growth on solid and in liquid media and a higher optimal growth temperature $\left(37^{\circ} \mathrm{C}\right)$ than previously described North America lymph node isolates (Appendix Figure 3). CGD mice infected with CSF strain showed higher plasma interleukin- $1 \beta$, tumor necrosis factor- $\alpha$, and interleukin- 6 than those infected with type strain 4 and 8 weeks after infection, which correlated with differences in tissue bacterial load. Cytokine levels did not increase in wild-type mice infected with CSF strain (Appendix Figure 4).

\section{Conclusions}

G. bethesdensis is an emerging pathogen shown to cause infection exclusively in CGD patients and has a spectrum of disease severity ranging from chronic and recurrent infections to fulminant sepsis, central nervous system infection, and death $(3,7,10)$. Until recently, all reported North America cases were nonfatal chronic infections; 1 case from Europe (Spain) was fatal. Recently, Mayer et al. reported an X-linked CGD patient in the United States who died of fulminant infection with an organism with $100 \%$ identity to $500 \mathrm{bp}$ of $G$. bethesdensis $16 \mathrm{~S}$ (11). Unfortunately, that $G$. bethesdensis isolate was not available for analysis and comparison with other $G$. bethesdensis strains. The previous strain from Europe was highly resistant to antimicrobial agents, including colistin, most $\beta$-lactams, and quinolones (10).

We found that a CSF $G$. bethesdensis strain, showing an identical $16 \mathrm{~S}$ sequence to a previously described fulminant strain from Europe, was more virulent and lethal in a mouse model than the $G$. bethesdensis US type strain and more virulent in gp91 ${ }^{\text {phox-/- }}$ than in $\mathrm{p} 47^{\text {phox }-/-}$ mice. A fatal case of $G$. bethesdensis infection in the United States suggests that heterogeneity might exist among North America $G$. bethesdensis strains. Bacterial genome sequencing may identify discrete virulence factors. $G$. bethesdensis must be included as a cause of fatal disseminated infection in CGD.

This work was supported through the Division of Intramural Research, National Institute of Allergy and Infectious Diseases, National Institutes of Health. 


\section{About the Author}

Dr. Rebelo is a pediatrics resident in Hospital Dona Estefânia, Centro Hospitalar de Lisboa Central. Her primary research interests are clinical immunology, primary immunodeficiencies, and infectious diseases

\section{References}

1. Holland SM. Chronic granulomatous disease. Hematol Oncol Clin North Am. 2013;27:89-99, viii. http://dx.doi.org/10.1016/ j.hoc. 2012.11 .002

2. Gennery A. Recent advances in understanding and treating chronic granulomatous disease. F1000 Res. 2017;6:1427. http://dx.doi.org/10.12688/f1000research.11789.1

3. Falcone EL, Petts JR, Fasano MB, Ford B, Nauseef WM, Neves JF, et al. Methylotroph infections and chronic granulomatous disease. Emerg Infect Dis. 2016;22:404-9. http://dx.doi.org/10.3201/ eid2203.151265

4. Greenberg DE, Ding L, Zelazny AM, Stock F, Wong A, Anderson VL, et al. A novel bacterium associated with lymphadenitis in a patient with chronic granulomatous disease. PLoS Pathog. 2006;2:e28. http://dx.doi.org/10.1371/journal.ppat.0020028

5. Greenberg DE, Porcella SF, Stock F, Wong A, Conville PS, Murray PR, et al. Granulibacter bethesdensis gen. nov., sp. nov., a distinctive pathogenic acetic acid bacterium in the family Acetobacteraceae. Int J Syst Evol Microbiol. 2006;56:2609-16. http://dx.doi.org/10.1099/ijs.0.64412-0

6. Greenberg DE, Porcella SF, Zelazny AM, Virtaneva K, Sturdevant DE, Kupko JJ III, et al. Genome sequence analysis of the emerging human pathogenic acetic acid bacterium Granulibacter bethesdensis. J Bacteriol. 2007;189:8727-36. http://dx.doi.org/10.1128/JB.00793-07

7. Greenberg DE, Shoffner AR, Zelazny AM, Fenster ME, Zarember KA, Stock F, et al. Recurrent Granulibacter bethesdensis infections and chronic granulomatous disease. Emerg Infect Dis. 2010;16:1341-8. http://dx.doi.org/10.3201/ eid1609.091800

8. Zarember KA, Marshall-Batty KR, Cruz AR, Chu J, Fenster ME, Shoffner AR, et al. Innate immunity against Granulibacter bethesdensis, an emerging gram-negative bacterial pathogen. Infect Immun. 2012;80:975-81. http://dx.doi.org/10.1128/IAI.05557-11

9. Chu J, Song HH, Zarember KA, Mills TA, Gallin JI. Persistence of the bacterial pathogen Granulibacter bethesdensis in chronic granulomatous disease monocytes and macrophages lacking a functional NADPH oxidase. J Immunol. 2013;191:3297-307. http://dx.doi.org/10.4049/jimmunol.1300200

10. López FC, de Luna FF, Delgado MC, de la Rosa II, Valdezate S, Nieto JA, et al. Granulibacter bethesdensis isolated in a child patient with chronic granulomatous disease. J Infect. 2008; 57:275-7. http://dx.doi.org/10.1016/j.jinf.2008.04.011

11. Mayer EFF, Gialanella P, Munjal I, Cunningham-Rundles C, Dara J. Fulminant sepsis due to Granulibacter bethesdensis in a 4-year-old boy with X-linked chronic granulomatous disease. Pediatr Infect Dis J. 2017;36:1165-6. http://dx.doi.org/10.1097/ INF.0000000000001659

Address for correspondence: João Farela Neves, Primary Immunodeficiencies Unit, Hospital Dona Estefânia, Rua Jacinta Marto, 1145, Lisbon, Portugal; email: joao.farelaneves@chlc.min-saude.pt

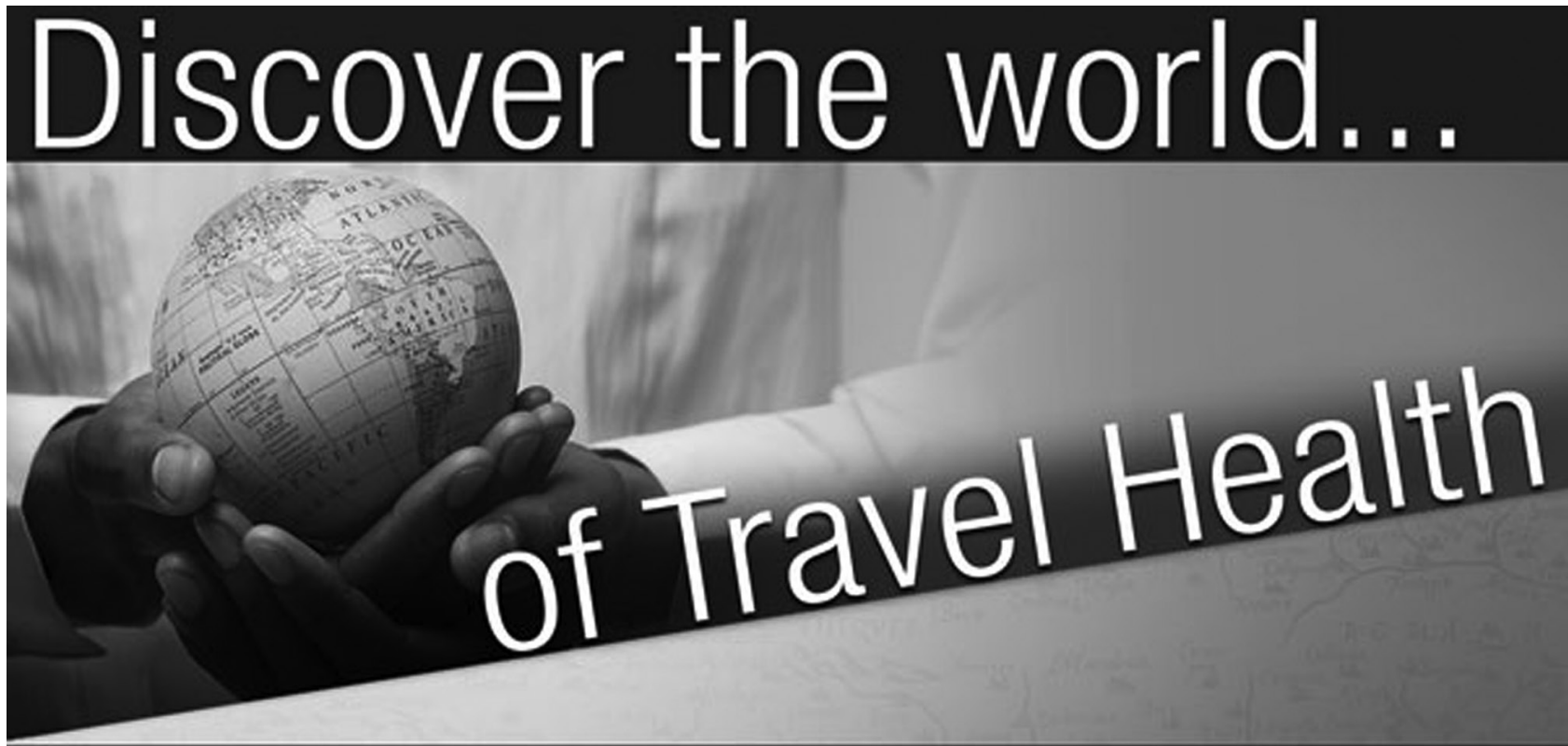

\section{www.cdc.gov/travel}

Visit the CDC Travelers' Health website for up-to-date information on global disease activity and international travel health recommendations.

Department of Health and Human Services - Centers for Disease Control and Prevention 